(2) The Editor of the Referativny Zurnal, Astronomija i Geodesia, Moskva D-2rg Baltijskij Pos. D. 42B.

(3) L'éditeur du Bulletin Analytique du C.N.R.S., 45 rue d'Ulm, Paris-V, France.

(4) L'éditeur des Astronomical Newes Letters, Laboratoire d'astronomie, Porte de Douai, Lille (Nord), France.

2. La Commission 5 prie l'U.A.I. de prévoir pour la prochaine période de trois ans, annuellement, une somme de 500 dollars (crédit total 4500 dollars) de manière à assurer la continuation de la publication des Astronomical News Letters sous la direction du Dr V. Kourganoff du Laboratoire d'astronomie de l'Université de Lille. Il est entendu que le Dr Kourganoff, d'accord avec le président de la Commission 5, préparera une liste des instituts astronomiques qui recevront gratuitement les $A . N . L$. Le nombre total des instituts qui recevront de cette manière les $A$.N.L. ne dépassera pas, dans le cadre du crédit prévu ci-dessus, deux cents.

3. La Commission témoigne sa satisfaction quant à l'état d'avancement du travail entrepris pour combler la lacune entre la Bibliographie générale de l'astronomie de Houzeau et Lancaster et l'A stronomischer Jahresbericht, et insiste pourque les dispositions nécessaires soient prises pour terminer le manuscrit avant la prochaine Assemblée générale de l'U.A.I.

4. La Commission, appréciant l'étendue de la diffusion donnée aux Extraits d'astronomie $d u$ Bulletin analytique du C.N.R.S., estime que la subvention qui a été donnée à celui-ci doit être renouvelée à concurrence de $I_{5} 00$ francs-or pour la période des trois années à venir (1956-57-58).

5. La Commission, ayant pris connaissance de l'édition provisoire du Répertoire: Les observatoires astronomiques et les astronomes, et heureuse des résultats atteints, estime qu'il y aura lieu d'attribuer à l'Observatoire royal de Belgique une seconde et dernière subvention de 500 dollars pour permettre d'entreprendre l'édition définitive de cet ouvrage.

\title{
Commission 6
}

La Commission demande à l'U.A.I. de continuer sa subvention de I200 francs-or par an au Bureau Central des Télégrammes Astronomiques.

\section{Commission 8}

I. La Commission approuve unanimement les recommandations de la Conférence d'Astrométrie de Bruxelles du 28-30 mars I955. Ces recommandations ont été imprimées dans les Communications de l'Observatoire royal de Belgique, no. 85, pp. 37-42.

Les observatoires de Babelsberg et Ottawa ont été ajoutés à la liste primitive (p. 38) des observatoires qui prendront part aux observations méridiennes.

Le comité prévu au Nr. VII des Résolutions (p. 39) sera constitué par les spécialistes suivants: F. P. Scott (président), D. Brouwer, A. DANjon, W. Fricke, O. Heckmann, A. Nemiro, L. S. T. Symms, M. S. Zverev.

2. La Commission recommande que dans tous les catalogues méridiens, outre l'époque de l'observation pour chaque étoile soit donné au moins: le Temps Moyen d'Observation dans l'Année pour l'ensemble des observations de l'étoile; il serait préférable de donner les facteurs parallactiques en $a$ et $\delta$, si les dates des observations avaient une repartition défavorable.

3. L'Union Astronomique Internationale appelle l'attention sur la nécessité de nouvelles observations méridiennes dans l'hémisphère austral, et elle insiste auprès des gouvernements de l'Australie et de l'Afrique Australe pour que les crédits indispensables à la mise en service des instruments existants soient accordés.

\section{Commission 9}

Les convertisseurs d'images photoélectriques ont atteint un point de leur développement tel que leur supériorité sur les méthodes photographiques devient évidente en de 
nombreux domaines. Vu l'importance de la coopération de l'industrie dans la fabrication et la diffusion des systèmes de convertisseurs d'images à usage astronomique, l'Union Astronomique Internationale peut et devrait user de son influence et prestige dans ce problème. Il est donc souhaitable que l'importance des convertisseurs d'images et leurs applications à l'astronomie soient reconnues et trouvent une place dans l'activité de l'Union.

En terms plus précis, le rôle initial d'une sous-commission concernant les convertisseurs d'images photoélectriques pourrait être: l'encouragement d'une fabrication commerciale des élements dont dépend la généralisation de ces méthodes, la normalisation des charactéristiques d'adaptation aux instruments astronomiques et aux spécifications des appareils commerciaux, l'obtention des facilités vis-à-vis des brevets et licenses en ce qui concerne les applications astronomiques, et l'encouragement des recherches ultérieures.

Nous souhaiterions donc que l'Union veuille bien approuver l'établissement d'une sous-commission concernant les Convertisseurs d'Images au sein de la Commission 9 (Instruments). Le président par interim a marqué son accord à ce sujet. Nous suggérons que la sous-commission concernant les Convertisseurs d'Images comprenne les membres suivants, qui travaillent dans ce domaine: BAum (président), Duchesne, FellgetT, J. S. Hall, Hiltner, Krassovsky, Lallemand, Leighton, McGee, Morton, and A. G. Wilson.

\section{Commission ro}

I. La Commission recommande la mise au point précise, à l'occasion de l'Année Géophysique Internationale, de l'étude détaillée en commun de l'évolution des taches solaires, sous la direction de l'Observatoire fédéral de Zurich, conformément à la résolution adoptée par la septième Assemblée générale de l'U.A.I. en I948.

2. La Commission recommande que la subvention annuelle de $x 000$ francs-or, accordée précédemment à l'Observatoire de Zurich pour la publication des Cartes héliographiques de la Photosphère, soit renouvelée pour la période qui s'étendra jusqu'à la prochaine Assemblée générale.

3. La Commission recommande qu'une subvention annuelle de 1500 dollars soit accordée à l'Observatoire de Zurich pour la publication du Quarterly Bulletin on Solar Activity, pendant la période qui s'écoulera jusqu'à la prochaine Assemblée générale.

\section{Commission II}

I. La Commission recommande que la subvention annuelle de 2700 francs-or, accordée depuis I925 par l'U.A.I. à l'Observatoire de Meudon pour la publication des Cartes synoptiques de la Chromosphère solaire, soit renouvelée pour la période qui s'écoulera jusqu'à la prochaine Assemblée générale.

2. La Commission recommande que le nouveau mode de classification des éruptions solaires tel qu'il est présenté dans la Section 3 (page I53 de ce volume), soit adopté à partir du premier janvier 1956.

\section{Commission $\mathbf{I 2}$}

I. La Commission recommande instamment à tous les laboratoires de physique et tous les laboratoires d'Astrophysique convenablement outillés la détermination des 'forces d'oscillateur' (valeurs de $f$ ) et autres constantes astrophysiques dont les astronomes ont un besoin urgent.

2. La Commission recommande instamment l'affectation d'un crédit de 2000 dollars à la publication des Tables spectroscopiques solaires (Solar Spectroscopic Tables), dont on prévoit l'achèvement avant la prochaine Assemblée de l'U.A.I. en I958.

Ces tables sont d'une importance capitale pour tous les travaux de spectroscopie solaire et stellaire. 